\title{
Evidence for gating of direct response activation in the Simon task
}

\author{
PETER WÜHR \\ Friedrich-Alexander Universität, Erlangen, Germany
}

\begin{abstract}
The Simon effect denotes faster responses when the task-irrelevant stimulus position corresponds to the response position than when it does not. Accounts of this effect assume that stimulus position automatically activates a spatially corresponding response while the correct response is being computed. Yet the Simon effect has been found to be reduced after noncorresponding trials. Some authors have interpreted these sequential modulations of the Simon effect as evidence for a mechanism gating positionbased response activation. Alternatively, sequential modulations have been explained in terms of featureintegration processes, which depend upon the fact that different sequences of spatial-correspondence conditions covary with different degrees of feature overlap between subsequent trials. The present study investigates whether sequential modulations of the Simon effect can occur when feature overlap in the different conditions is the same. Therefore, a Simon task with four stimulus positions and two response positions was used. Sequential modulations of the Simon effect were found in trial sequences with constant amounts of feature overlap between trials. Although the feature-integration account cannot explain this result, it is consistent with the idea of a gating (i.e., cognitive control) mechanism.
\end{abstract}

The spatial correspondence between stimuli and responses affects performance even when stimulus position is irrelevant for the task at hand. Consider a task in which left responses are mapped onto green stimuli and right responses are mapped onto red stimuli, while stimuli appear randomly to the left or to the right. Again, spatially corresponding S-R conditions (e.g., green stimuli appearing to the left) produce better performance than do spatially noncorresponding S-R conditions (e.g., green stimuli appearing to the right; Simon \& Berbaum, 1990). This observation is called the Simon effect (for a review, see Lu \& Proctor, 1995). The Simon effect is not restricted to the horizontal dimension; it also occurs when stimuli and responses vary on the vertical axis (e.g., Stürmer, Leuthold, Soetens, Schröter, \& Sommer, 2002).

The dominating accounts of the Simon effect distinguish two parallel routes of response activation in the Simon task (e.g., Hommel, 1997; Zhang, Zhang, \& Kornblum, 1999). An indirect route processes the relevant (i.e., nonspatial) stimulus feature and activates the correct response in accordance with task instructions. A direct route processes the irrelevant stimulus position and activates the spatially corresponding member of the response set. In spatially corresponding conditions, both pathways activate one and the same response, which is quickly selected and executed. In contrast, in noncorre-

I thank Jessica Bogner for collecting the data and Bernhard Hommel, Hartmut Leuthold, Toby Mordkoff, and an anonymous reviewer for insightful comments on an earlier draft. Correspondence concerning this article should be addressed to P. Wühr, Institut für Psychologie I, FriedrichAlexander Universität, Kochstrasse 4, 91054 Erlangen, Germany (e-mail: prwuehr@phil.uni-erlangen.de). sponding conditions, the pathways activate different responses, and a response conflict has to be resolved.

Position-based response activation through the direct route has often been described as automatic (e.g., Zhang et al., 1999). Correspondingly, several authors assumed that direct response activation rests on strong associations between spatial stimulus codes and spatial response codes in long-term memory (e.g., Barber \& O'Leary, 1997). Such a process might be expected to affect behavior almost inevitably, yet this is not the case. For example, the occurrence of the Simon effect in a given trial has been found to depend upon spatial S-R correspondence in the preceding trial. In particular, a large Simon effect was observed after corresponding trials. After noncorresponding trials, however, the Simon effect was strongly reduced (e.g., Praamstra, Kleine, \& Schnitzler, 1999; Ridderinkhof, 2002) or absent (e.g., Stürmer et al., 2002; Valle-Inclán, Hackley, \& de Labra, 2002; Wühr, 2004). This observation is important because it challenges a central claim of many dual-route accounts of the Simon effect (cf. Hommel, Proctor, \& Vu, 2004; Notebaert, Soetens, \& Melis, 2001).

To explain sequential modulations of the Simon effect, some authors have proposed the existence of mechanisms gating position-based response activation in the Simon task (e.g., Ridderinkhof, 2002; Stürmer et al., 2002). For example, Stürmer et al. proposed an "ancillary monitoring mechanism" (AMM) that modulates the impact of position-based response activation upon the motor system. Stürmer et al. described two ways in which the AMM might work: It might close the direct route after each noncorresponding trial, or it might open the direct route after each corresponding trial. Interestingly, a re- 
cent study found Simon effects after neutral trials to be of intermediate size, when compared with Simon effects after corresponding and noncorresponding trials (Wühr \& Ansorge, 2005). This result suggests that the AMM, if such a mechanism exists at all, might increase the conductivity of the direct route after corresponding trials and decrease it after noncorresponding trials. In the following, I refer to these ideas as gating accounts. ${ }^{1}$

An alternative explanation for sequential modulations of the Simon effect rests on the concept of event files (Hommel, 1998). The basic idea is that when stimuli and responses co-occur in time, their features are spontaneously encoded into a common, short-lived representation. This representation is called an event file (Hommel, 1998). Yet the cognitive codes of stimulus and response features cannot be used for an infinite number of event files simultaneously (Stoet \& Hommel, 1999). Imagine, for example, a situation in which an event file representing a yellow square and a right keypress already exists when a second event file is to be formed in order to represent a red square and a right keypress. In this situation, the feature code RIGHT must be "unbound" from the first event file before it can be integrated into the second one. This unbinding process is assumed to take time. In contrast, event-file formation (i.e., feature integration) is assumed to proceed normally if either all or none of the needed codes are part of already existing event files.

Importantly, in the usual Simon task the amount of feature overlap between two subsequent S-R episodes (i.e., trials) is confounded with the sequence of spatial S-R correspondence conditions (cf. Hommel et al., 2004; Notebaert et al., 2001). In the typical two-choice task (two stimulus colors, two stimulus positions, two response positions), the 16 possible first-order trial sequences can be divided into four corresponding-corresponding $(\mathrm{Co}-\mathrm{Co})$, four corresponding-noncorresponding $(\mathrm{Co}-\mathrm{Nc})$, four noncorresponding-corresponding $(\mathrm{Nc}-\mathrm{Co})$, and four noncorresponding-noncorresponding $(\mathrm{Nc}-\mathrm{Nc})$ sequences. Importantly, in $\mathrm{Co}-\mathrm{Co}$ sequences and in $\mathrm{Nc}-\mathrm{Nc}$ sequences either all features repeat (complete repetition) or all features alternate (complete alternation). In contrast, in $\mathrm{Co}-\mathrm{Nc}$ sequences and in Nc-Co sequences some features repeat, whereas others alternate (partial repetitions or partial alternations).

The feature-integration account explains sequential modulations of the Simon effect in the following way. In $\mathrm{Co}-\mathrm{Co}$ sequences, either all or none of the features are repeated, and event-file formation proceeds normally. Moreover, spatial S-R correspondence aids performance in trial N. In contrast, all $\mathrm{Co}-\mathrm{Nc}$ sequences comprise partial repetitions. This fact slows feature integration in trial $\mathrm{N}$, and spatial S-R noncorrespondence also impairs performance. In other words, for Co-Nc sequences feature integration and spatial S-R correspondence work in the same direction, increasing the Simon effect after corresponding trials. In contrast, for $\mathrm{Nc}-\mathrm{Co}$ sequences feature integration and spatial S-R correspondence work in opposite directions, decreasing the Simon effect after noncorresponding trials (cf. Hommel et al., 2004; Valle-Inclán et al., 2002). Recently, Hommel et al. demonstrated that sequential modulations of the Simon effect could occur when stimulusinduced response conflicts in the preceding trial were minimized or excluded, an observation that supports the feature-integration account. Yet these data do not rule out the possibility that a gating mechanism might also produce sequential modulations of the Simon effect.

The present study investigated whether sequential modulations of the Simon effect can occur when featureintegration effects are held constant across the different experimental conditions. Such a situation is provided by a variant of the Simon task with two stimulus colors, four stimulus positions, and two response positions. Note that participants have the same task as usual: They press a lower key in response to green stimuli and an upper key in response to red stimuli, or vice versa. The only difference is that the stimulus can appear at four positions (lower left, upper left, upper right, lower right), rather than two positions. As a result, 64 first-order trial sequences are possible (cf. the Appendix). Accordingly, there are $16 \mathrm{Co}-\mathrm{Co}$ sequences, $16 \mathrm{Co}-\mathrm{Nc}$ sequences, 16 $\mathrm{Nc}-\mathrm{Co}$ sequences, and $16 \mathrm{Nc}-\mathrm{Nc}$ sequences. Importantly, in this task, trial sequences with the same amount of feature overlap can be found among each of the four sequences of correspondence conditions. In particular, for each correspondence sequence there are cases for which stimulus color and response position repeat while stimulus position changes (partial repetitions), and there are cases for which everything changes (complete alternation). In contrast, complete repetitions and partial alternations (stimulus color and response alternate while stimulus position repeats) do not occur among each correspondence sequence (cf. the Appendix). Therefore, the present study mainly focused on partial repetitions and complete alternations.

The feature-integration account does not predict sequential modulations of the Simon effect when only trial sequences of the same feature-transition type are compared. When only partial-repetition sequences are compared, unbinding costs should occur in every trial, and therefore they cannot differentially affect $\mathrm{S}-\mathrm{R}$ correspondence in trial N. Similarly, unbinding costs should never occur when only complete alternations are compared. Thus, observing sequential modulations of the Simon effect under these conditions would suggest that feature-integration effects are not the only possible source of sequential modulations. Moreover, such an observation could be taken as evidence for gating of direct response activation in the Simon task. Yet the validity of this conclusion depends upon both the vertical and horizontal dimensions being processed. Processing of the vertical dimension can be expected, because responses vary on that dimension, for which previous studies have established Simon effects (e.g., Stürmer et al., 2002). However, the horizontal dimension is completely irrelevant for the present task and, hence, processing of this dimension cannot be taken for granted (cf. Hommel, 
1996). Fortunately, processing of the horizontal dimension can be assessed by comparing performance for complete repetitions of displays with performance for sequences that are almost identical to the complete repetitions, except that horizontal stimulus position had changed between trials. Better performance in the former condition would indicate that the horizontal dimension was processed.

\section{Method}

Participants. Nineteen students (17 female, mean age: 22 years) participated in a single-session experiment. The experiment lasted about $45 \mathrm{~min}$, and participants were paid $5 \square$. All participants were naive with respect to the purpose of the study and classified themselves as having normal (or corrected-to-normal) visual acuity.

Apparatus and Stimuli. The participants sat in front of a 17-in. color monitor, with unconstrained viewing distance of approximately $50 \mathrm{~cm}$. An IBM-compatible computer controlled the presentation of visual stimuli, which were shown on a black background. The fixation point was a small "+" sign $\left(\sim 0.3^{\circ}\right)$. Stimuli were filled rectangles with a side length of $16 \mathrm{~mm}\left(\sim 1.8^{\circ}\right)$, appearing either in red or green at one of four positions. Stimulus positions were to the lower left, upper left, upper right, or lower right of the screen center. At each position, the distance between the inner edge of the stimulus and the screen center was $44 \mathrm{~mm}\left(\sim 5.0^{\circ}\right)$. Two response buttons were mounted above each other on a wooden rack, with a distance of $15 \mathrm{~cm}$ between buttons. The buttons were connected to the computer via the Exkey interface device (Berisoft Corp., Frankfurt/Main, Germany). The participants operated the lower key with their left hand and the upper key with their right hand for half of the experiment, whereas the opposite held for the remaining half of the experiment.

Procedure. The experiment began with the presentation of the instructions on the screen. They described the stimuli and the sequence of events in a typical trial. Moreover, the instructions informed the participants about the mapping of stimulus colors onto response keys. Then, the participants practiced their task in a block of 24 test trials.

The experimental phase contained 10 blocks of 64 trials, each of which contained the following sequence of events. After a blank period of $500 \mathrm{msec}$, the fixation point appeared at screen center and remained on the screen. Next, $500 \mathrm{msec}$ after the onset of the fixation point, a red or green rectangle appeared for $250 \mathrm{msec}$ at one of four possible positions (see above). The participants were instructed to respond to stimulus color by pressing the appropriate key as quickly as possible. Ten participants pressed the lower key to green stimuli and the upper key to red stimuli; 9 participants received the opposite instructions. Stimulus presentation was followed by a 750msec blank period.

For error-free trials, the stimulus onset asynchrony between subsequent stimuli was $2 \mathrm{sec}$. However, when the participants pressed the wrong key or reaction time (RT) exceeded $1 \mathrm{sec}$, a corresponding error message was shown for $1 \mathrm{sec}$ more. After each block, the participants could take a rest before starting the next block at their leisure.

Design. The experiment rested on a $2 \times 2$ within-subjects design. The first factor was preceding $\mathrm{S}-\mathrm{R}$ correspondence $(\mathrm{S}-\mathrm{R}$ correspondence in trial $\mathrm{N}-1$ ). The second factor was $\mathrm{S}-\mathrm{R}$ correspondence in the present trial ( $\mathrm{S}-\mathrm{R}$ correspondence in trial $\mathrm{N})$. To investigate the main question of the present study, separate analyses were planned for complete alternations and for partial repetitions (cf. the Appendix). In each block, the participants received eight repetitions of each combination of two stimulus colors and four stimulus positions in random order.

\section{Results}

For each participant, I first removed all RTs exceeding two standard deviations from the grand mean (cf. Van Selst
\& Jolicœur, 1994). Across participants, RTs $<375 \mathrm{msec}$ $(1.3 \%)$ and RTs $>793 \mathrm{msec}(2.8 \%)$ were excluded from further analyses. Moreover, only RTs from error-free trials that were preceded by an error-free trial were analyzed.

Reaction times. First, a one-factorial analysis of variance (ANOVA) was conducted on the complete data set, with sequence type (complete repetition, partial repetition, partial alternation, complete alternation) as the within-subjects factor. There was a significant effect of sequence type $[F(3,54)=9.71, p<.001]$. RTs were shorter for complete repetitions $(571 \mathrm{msec})$ than for partial repetitions [588 msec; $t(18)=5.21, p<.001]$ and partial alternations [597 msec; $t(18)=4.38, p<.001$ ]. In contrast, complete repetitions were not different from complete alternations [580 msec; $t(18)=1.68, p=.11$, and partial repetitions were not different from partial alternations $[t(18)=1.60, p=.13]$. This pattern of results is consistent with the feature-integration account (cf. Hommel et al., 2004).

Next, separate ANOVAs were performed on RTs from partial repetitions and on RTs from complete alternations (cf. Figure 1, upper panel). For partial repetitions, the main effect for preceding correspondence was not significant $[F(1,18)=1.85, p=.191]$. Yet a significant main effect of present correspondence $[F(1,18)=66.14$, $p<.001]$ indicated shorter RTs for corresponding conditions $(575 \mathrm{msec})$ than for noncorresponding conditions $(597 \mathrm{msec})$. Moreover, the significant interaction indicated sequential modulation of the Simon effect $[F(1,18)=$ 21.62, $p<.001]$. There was a larger Simon effect after corresponding trials $(33 \mathrm{msec})$ than after noncorresponding trials $(12 \mathrm{msec})$. For complete alternations, the main effect for preceding correspondence approached significance $[F(1,18)=3.31, p=.086]$, suggesting somewhat shorter RTs after corresponding trials $(579 \mathrm{msec})$ than after noncorresponding trials $(583 \mathrm{msec})$. A significant main effect of present correspondence $[F(1,18)=60.69$, $p<.001]$ indicated shorter RTs for corresponding conditions $(570 \mathrm{msec})$ than for noncorresponding conditions $(593 \mathrm{msec})$. Moreover, the significant interaction indicated sequential modulation of the Simon effect $[F(1,18)=$ $5.86, p<.05]$. There was a larger Simon effect after corresponding trials $(29 \mathrm{msec})$ than after noncorresponding trials $(18 \mathrm{msec})$.

The impact of changes on the irrelevant horizontal dimension between trials was assessed by comparing RTs for complete repetitions of displays (e.g., Co1-Co1; see the Appendix) with RTs for display sequences that are almost identical to the complete repetitions, except for a change of horizontal stimulus position between trials (e.g., Co1-Co2; cf. the Appendix). Shorter RTs for complete repetitions $(571 \mathrm{msec})$ than for changes of horizontal stimulus position [581 msec; $F(1,18)=6.61, p<.05$ ], suggests that the horizontal dimension was processed.

Error percentages. First, a one-factorial ANOVA was conducted on the complete data set, with sequence type as the within-subjects factor. The effect of this variable was significant $[F(3,54)=9.14, p<.001]$. This result indicates fewer errors for complete repetitions $(1.8 \%)$ 

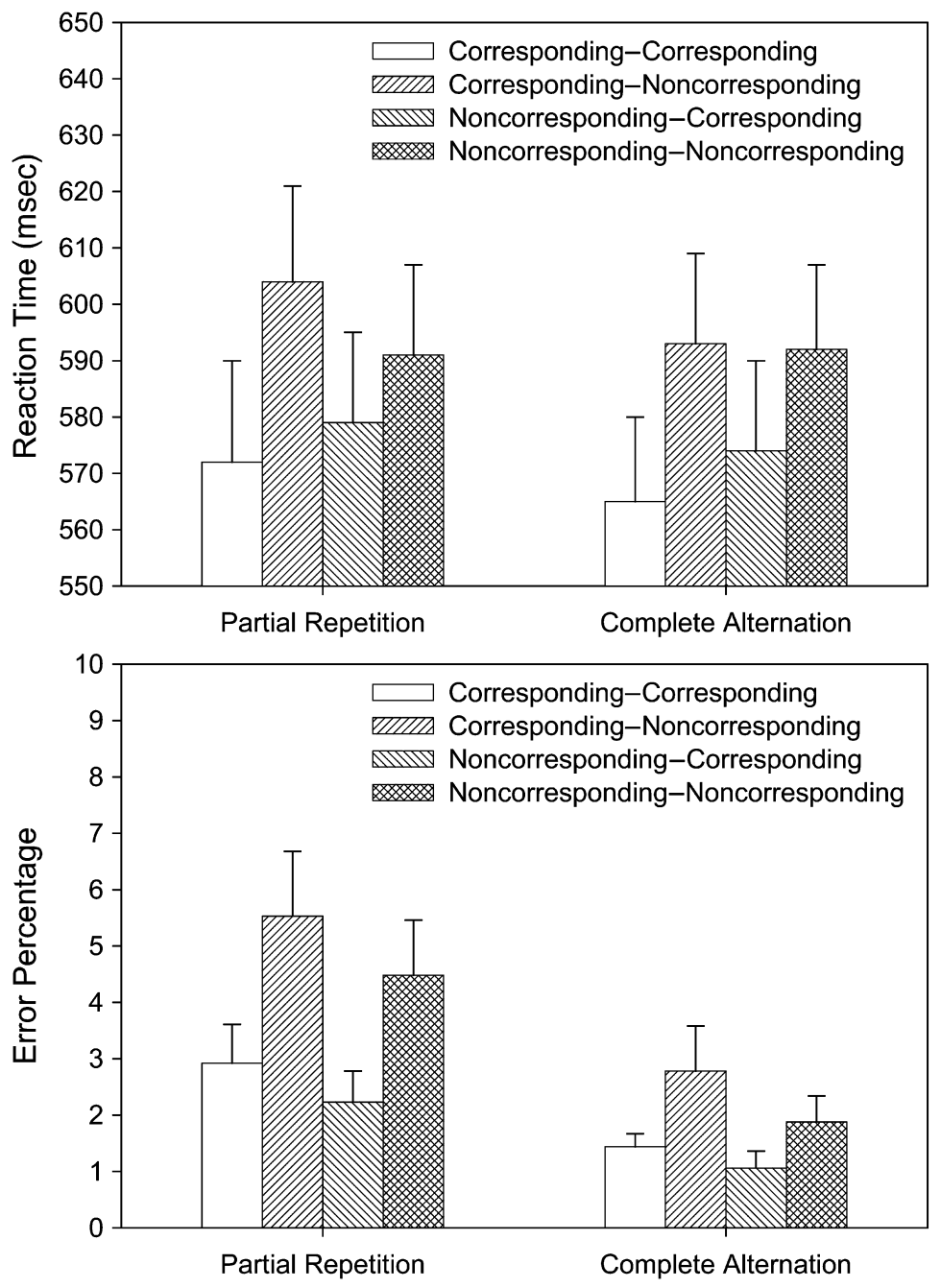

Feature Transition

Figure 1. Reaction times (upper panel) and error percentages (lower panel) observed in the present experiment, as a function of correspondence-condition sequence and of feature transition between subsequent trials. Error bars represent standard errors of the mean between participants $(N=19)$.

and complete alternations $(1.7 \%)$ than for partial repetitions $(3.9 \%)$ and partial alternations $(3.1 \%)$.

Next, separate ANOVAs were performed on error percentages for the two types of trial sequences (cf. Figure 1 , lower panel). The only significant results involved the main effects of present correspondence, indicating a Simon effect of $2.4 \%$ for partial repetitions $[F(1,18)=$ $15.32, p<.01]$ and a Simon effect of $1.1 \%$ for complete alternations $[F(1,18)=4.49, p<.05]$. Moreover, I conducted a comparison between error rates for complete repetitions and error rates for similar display sequences, in which only the horizontal stimulus position had changed. A lower error percentage for complete repetitions (1.7\%) than for changes on the horizontal dimension $[3.7 \%$; $F(1,18)=11.68, p<.01]$ indicates that the horizontal dimension was processed.

\section{Discussion}

The present study investigates whether sequential modulations of the Simon effect can occur when the feature overlap between subsequent trials is the same in each of the four possible sequences of spatial-correspondence conditions. The experiment involved a variant of the Simon task with four stimulus positions and two response positions. In this task, one finds trial sequences with the same degree of feature overlap among each of the four sequences of spatial-correspondence conditions. First, there are sequences in which stimulus color and response repeat while stimulus position alternates (partial repetitions) in each condition. Second, there are also sequences with complete alternations in each condition. The present experiment revealed sequential modulations of the Simon effect for both types of trial sequences. Yet 
before these results can be interpreted, we must assess whether participants processed horizontal stimulus position along with vertical stimulus position. Otherwise, we cannot assume that complete alternations and partial repetitions were among each of the four sequences of spatial-correspondence conditions. A comparison of performance in trial sequences involving complete repetitions of stimulus displays with performance in similar trial sequences, except for a change of horizontal stimulus position between trials, revealed shorter RTs and higher accuracy for the former condition. This result suggests that participants processed horizontal stimulus position along with vertical stimulus position (cf. Vu \& Proctor, 2002). Therefore, feature-integration processes cannot explain the modulations of the Simon effect observed in the present experiment. However, the results are consistent with the idea of a mechanism gating direct response activation. How might this mechanism work? Available evidence suggests that the gating mechanism (or AMM) is triggered by the correspondence and/or noncorrespondence between the response computed on the basis of color (i.e., the relevant stimulus feature) and the response activated by stimulus position (Wühr, 2004). Once triggered, the AMM might increase the conductivity of the direct route after corresponding trials and decrease it after noncorresponding trials (Wühr \& Ansorge, 2005).

It should be noted that a pilot experiment, in which responses varied on the horizontal dimension, revealed sequential modulations of the Simon effect for complete alternations only. The failure to obtain sequential modulations of Simon effects for partial repetitions with horizontally arranged responses may be related to a recent finding reported by Rubichi, Nicoletti, \& Umiltà (2005). These authors reported evidence suggesting that processing horizontal stimulus position provides a larger number of spatial codes than does processing vertical stimulus position. Due to the larger number of horizontal codes, it might be more difficult to suppress horizontal Simon effects than vertical Simon effects.

On first sight, the results of the present study seem at odds with those of the Hommel et al. (2004) study. Yet this is not the case. Hommel et al. showed that sequential modulations of the Simon effect could occur when variation of stimulus position is unlikely to affect response selection in the preceding trial. Importantly, however, this observation does not warrant concluding that interactions between processing stimulus position and selecting the correct response will never cause sequential modulations of the Simon effect. This conclusion would only be warranted if an impact of variable stimulus position upon response selection were demonstrated without simultaneously observing sequential modulations of the Simon effect in the subsequent trial. This demonstration is still lacking, however.

Together, the results reported by Hommel et al. (2004) and those of the present study suggest that at least two different mechanisms can cause sequential modulations of the Simon effect. One can be seen in the feature-integration processes that affect performance in Simon tasks, in addition to spatial S-R correspondence. A second cause for sequential modulations of the Simon effect may be a mechanism that gates direct response activation in the Simon task. Hommel et al. argued against this possibility that a gating mechanism should have to be rather sophisticated because it must know the different sources of response conflicts in order to be able to gate the source of unwanted activation. Yet determining the source of response activation might not be such a demanding task as suggested by Hommel et al. Besides that, it is also possible that the gating mechanism does not have to know the activation sources of incorrect responses at all. Note that, until now, most researchers assumed that, if a gating mechanism exists, it gates processing in the direct route. However, it is also possible that the gating mechanism modulates processing in the indirect (i.e., controlled) processing pathway. For example, the gating mechanism might increase the efficiency of processing in the controlled route after noncorresponding trials. Such a gating mechanism would be in perfect agreement with the assumption of dual-route models that positionbased response activation is automatic.

\section{REFERENCES}

BARber, P. J., \& O'LeAry, M. J. (1997). The relevance of salience: Towards an activational account of irrelevant stimulus-response compatibility effects. In B. Hommel \& W. Prinz (Eds.), Theoretical issues in stimulus-response compatibility (pp. 135-172). Amsterdam: Elsevier, North-Holland.

Botvinick, M. M., Braver, T. S., Barch, D. M., Carter, C. S., \& CoHen, J. D. (2001). Conflict monitoring and cognitive control. Psychological Review, 108, 624-652.

Gratton, G., Coles, M. G. H., \& Donchin, E. (1992). Optimizing the use of information: Strategic control of activation of responses. Journal of Experimental Psychology: General, 121, 480-506.

Hommel, B. (1996). No prevalence of right-left over top-bottom spatial codes. Perception \& Psychophysics, 58, 102-110.

Hommel, B. (1997). Toward an action-concept model of stimulus-response compatibility. In B. Hommel \& W. Prinz (Eds.), Theoretical issues in stimulus-response compatibility (pp. 281-320). Amsterdam: Elsevier, North-Holland.

Hommel, B. (1998). Event files: Evidence for automatic integration of stimulus-response episodes. Visual Cognition, 5, 183-216.

Hommel, B., Proctor, R. W., \& Vu, K.-P. L. (2004). A featureintegration account of sequential effects in the Simon task. Psychological Research, 68, 1-17.

LU, C.-H., \& Proctor, R. W. (1995). The influence of irrelevant location information on performance: A review of the Simon and spatial Stroop effects. Psychonomic Bulletin \& Review, 2, 174-207.

Notebaert, W., Soetens, E., \& Melis, A. (2001). Sequential analysis of a Simon task: Evidence for an attention-shift account. Psychological Research, 65, 170-184.

PraAmstra, P., Kleine, B. U., \& Schnitzler, A. (1999). Magnetic stimulation of the dorsal premotor cortex modulates the Simon effect. NeuroReport, 10, 3671-3674.

RIDDERINKHOF, K. R. (2002). Activation and suppression in conflict tasks: Empirical classification through distributional analyses. In W. Prinz \& B. Hommel (Eds.), Attention and performance XIX: Common mechanisms in perception and action (pp. 494-519). Oxford: Oxford University Press.

Rubichi, S., Nicoletti, R., \& Umiltà, C. (2005). Right-left prevalence with task-irrelevant spatial codes. Psychological Research, 69, 167-178. 
Simon, J. R., \& Berbaum, K. (1990). Effect of conflicting cues on information processing: The "Stroop effect" vs. the "Simon effect." Acta Psychologica, 73, 159-170.

Stoet, G., \& Hommel, B. (1999). Action planning and the temporal binding of response codes. Journal of Experimental Psychology: Human Perception \& Performance, 25, 1625-1640.

Stürmer, B., Leuthold, H., Soetens, E., Schröter, H., \& SomMER, W. (2002). Control over location-based response activation in the Simon task: Behavioral and electrophysiological evidence. Journal of Experimental Psychology: Human Perception \& Performance, 28, 1345-1363.

Valle-Inclán, F., Hackley, S. A., \& De Labra, C. (2002). Does stimulus-driven response activation underlie the Simon effect? In W. Prinz \& B. Hommel (Eds.), Attention and performance XIX: Common mechanisms in perception and action (pp. 474-493). Oxford: Oxford University Press.

VAn Selst, M., \& Joliceur, P. (1994). A solution to the effect of sample size on outlier elimination. Quarterly Journal of Experimental Psychology, 47A, 631-650.
Vu, K.-P. L., \& Proctor, R. W. (2002). The prevalence effect in twodimensional stimulus-response compatibility is a function of the relative salience of the dimensions. Perception \& Psychophysics, 64, $815-828$.

WüHR, P. (2004). Sequential modulation of logical-recoding operations in the Simon task. Experimental Psychology, 51, 98-108.

WüHr, P., \& ANSORgE, U. (2005). Exploring trial-by-trial modulations of the Simon effect. Quarterly Journal of Experimental Psychology, 58A, 705-731.

Zhang, H., Zhang, J., \& Konnblum, S. (1999). A parallel distributed processing model of stimulus-stimulus and stimulus-response compatibility. Cognitive Psychology, 38, 386-432.

\section{NOTE}

1. Sequential modulations, similar to those discussed here, have also been observed for the flanker compatibility effect (e.g., Gratton, Coles, \& Donchin, 1992), and an explanation in terms of conflict monitoring has been proposed (cf. Botvinick, Braver, Barch, Carter, \& Cohen, 2001). 
APPENDIX

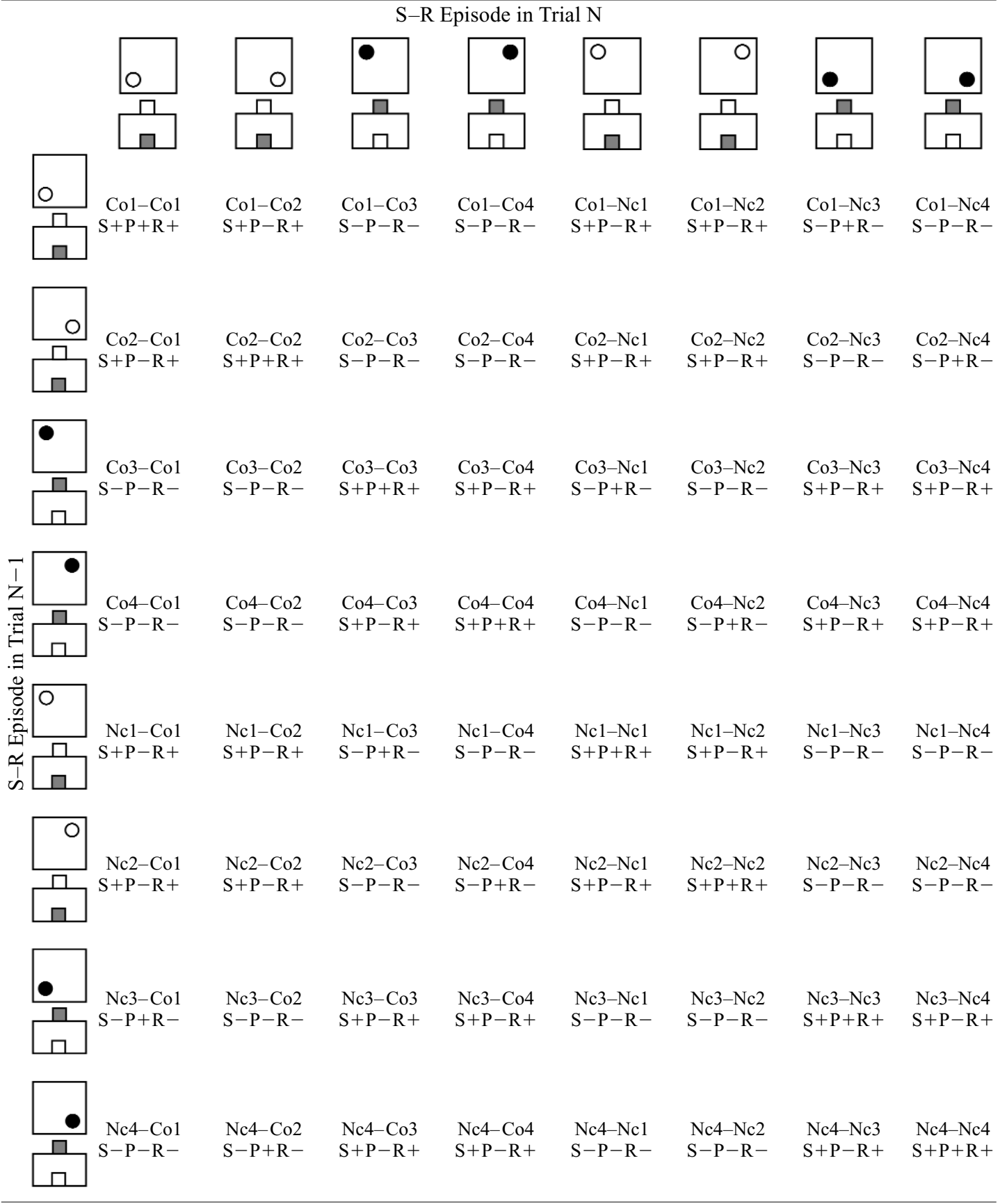

Note-Each cell of the table represents one of the possible 64 first-order trial sequences in the present experiment $(\mathrm{Co}=$ corresponding, $\mathrm{Nc}=$ noncorresponding, $\mathrm{S}=$ stimulus color, $\mathrm{P}=$ stimulus position, $\mathrm{R}=$ response, + denotes a repetition, - denotes an alternation). The stimulus-response mapping for the present example is "white circle—lower key, black circle—upper key."

(Manuscript received October 28, 2003;

revision accepted for publication June 18, 2004.) 